\title{
Eficiência de equações volumétricas para Manilkara spp. em floresta manejada na Amazônia Oriental
}

Misael Freitas dos SANTOS ${ }^{1 *}$, Afonso FIGUEIREDO FILHO ${ }^{1}$, João Ricardo Vasconcellos GAMA², Fabiane Aparecida de Souza RETSLAFF ${ }^{1}$, Daniele Lima da COSTA ${ }^{1}$, Karla Mayara Almada GOMES ${ }^{3}$, Girlene da Silva CRUZ ${ }^{4}$, Bruno Rafael Silva de ALMEIDA ${ }^{4}$

\author{
${ }^{1}$ Programa de Pós-Graduação em Ciências Florestais, Universidade Estadual do Centro-Oeste, Irati, PR, Brasil. \\ ${ }^{2}$ Programa de Pós-Graduação em Sociedade, Natureza e Desenvolvimento, Universidade Federal do Oeste do Pará, Santarém, PA, Brasil. \\ ${ }^{3}$ Laboratório de Manejo em Ecossistemas Florestais, Universidade Federal do Oeste do Pará, Santarém, PA, Brasil. \\ ${ }^{4}$ Programa de Pós-Graduação em Engenharia Florestal, Universidade do Estado de Santa Catarina, Lages, SC, Brasil. \\ *E-mail: freitasmta@gmail.com
}

Recebido em janeiro/2019; Aceito em abril/2019.

RESUMO: Objetivou-se avaliar a eficiência de equações específicas para Manilkara spp. em diferentes Unidades de Produção Anual (UPA) e em diferentes classes diamétricas, comparando-as com uma equação geral, em área florestal sob manejo na Floresta Nacional do Tapajós, Pará. Quatro modelos volumétricos foram ajustados, para um conjunto total de 5.231 árvores-amostras, a fim de obter-se uma equação geral representativa da espécie nas UPAs, bem como nas classes diamétricas nas quais as árvores foram distribuídas. O modelo selecionado foi ajustado por UPA e por classe diamétrica, obtendo-se diferentes equações específicas. O teste de identidade de modelos de Graybill foi aplicado para comparação das equações, e o teste Qui-quadrado foi utilizado para validação das mesmas. As equações específicas apresentaram desempenho superior quando comparadas à equação geral, tanto para as UPAs, quanto para as classes diamétricas. Além do mais, o teste de identidade mostrou haver diferença significativa entre tais equações, indicando que não é estatisticamente adequado utilizar uma única equação para as estimativas volumétricas de Manilkara spp. em todas as UPAs, bem como em todas as classes diamétricas. Portanto, recomenda-se a utilização de equações específicas, já que, além de serem válidas, as mesmas podem gerar estimativas mais precisas dos volumes comerciais da espécie.

Palavras-chave: espécie comercial nativa; identidade de modelos; estimativas de volume; manejo florestal na Amazônia; Floresta Nacional do Tapajós.

\section{Efficiency of volumetric equations for Manilkara spp. in managed forest in the Eastern Amazon}

\begin{abstract}
The objective of this study was to evaluate the efficiency of specific equations for Manilkara spp. in different Annual Production Units (UPA) and in different diametric classes, comparing them with a general equation, in the forest area under management in the Tapajós National Forest, Pará. Four volumetric models were adjusted for the total set of 5.231 sampled trees in order to obtain a representative general equation of the species in the UPAs as well as in the diametrical classes in which the trees were distributed. Then, the selected model was adjusted by UPA and by diameter class, obtaining different specific equations. The Graybill model identity test was applied to compare the equations, and the Chi-square test was used to validate the equations. The specific equations presented superior performance when compared to the general equation for the UPAs and for the diametric classes. In addition, the identity test showed a significant difference between the equations, indicating that it is not statistically adequate to use a single equation for the Manilkara spp. volumetric estimates in all UPAs and in all diametric classes. Therefore, we recommend using specific equations because, in addition to being valid, they can generate more accurate estimations of commercial volumes.
\end{abstract}

Keywords: native commercial species; model identity; volume estimates; forest management in the Amazon; Tapajós National Forest.

\section{INTRODUÇÃO}

$\mathrm{Na}$ Amazônia, a necessidade de quantificação do estoque de matéria prima florestal e a busca pela ótima produção e maior rendimento das florestas justificam a busca por ferramentas que possibilitem estimativas precisas do volume comercial das espécies e permitam a avaliação do potencial produtivo e econômico de determinada floresta (CYSNEIROS et al., 2017; THAINES et al., 2010; TONINI; BORGES, 2015).
Entretanto, as florestas tropicais apresentam como característica principal a abundância de espécies florestais, com as mais variadas dimensões, seja em função das distribuições diamétricas distintas, das alturas variadas ou das diferentes idades das árvores (FIGUEIREDO FILHO, 1983). Para este autor, isso afeta diretamente a forma das árvores e, consequentemente, dificulta o emprego dos métodos de estimativas, como as equações de volume, por exemplo. De acordo com Santos et al. (2012), nas florestas tropicais naturais, além da variabilidade ambiental, a grande 
heterogeneidade na composição das espécies, ou mesmo entre árvores de uma mesma espécie, torna-se um desafio na obtenção de equações de volume.

Sabe-se que o aprimoramento constante das técnicas de predição é de extrema importância na atividade florestal, uma vez que, como enfatizado por Gama et al. (2017), estimativas equivocadas dos estoques volumétricos ocasionam significativas perdas econômicas, visto que o planejamento ficará comprometido. Nesse contexto, o ajuste de equações volumétricas com base em conjuntos específicos de dados, como pequenas unidades de manejo ou classes diamétricas, que apresentem a menor variação possível, configura o procedimento mais eficiente, econômico e com precisão aceitável para a quantificação da produção em volume da floresta (BARROS; SILVA JÚNIOR, 2009).

Este procedimento se torna ainda mais importante quando considerada a importância comercial de determinadas espécies amazônicas como, por exemplo, a Manilkara spp., pertencente à família Sapotaceae e popularmente conhecida como maçaranduba.

A maçaranduba é uma das espécies mais valorizadas no mercado, por apresentar características como madeira muito pesada, dura, resistente, com densidade básica de $0,87 \mathrm{~g} \mathrm{~cm}^{-3}$ e de coloração escura, além de sua expressiva abundância na região (RIBEIRO, 2008; CASTRO; CARVALHO, 2014). De acordo com estes autores, tais características contribuem para que a espécie seja uma das mais exploradas e comercializadas na Amazônia, sendo utilizada em cerca de $90 \%$ das indústrias de transformação.

Entre os anos de 2005 e 2010, Manilkara spp. foi a espécie com maior número de árvores e, consequentemente, com maior volume de madeira extraído e comercializado na área de manejo florestal da Floresta Nacional do Tapajós (FNT), no estado do Pará. Assim, visando auxiliar a conservação da espécie por meio do manejo florestal sustentável, as atividades ligadas ao manejo da mesma devem ser aprimoradas como, por exemplo, por meio da obtenção de estimativas acuradas do estoque volumétrico, que sejam a base para o planejamento da atividade florestal.

Apesar da existência de estudos que disponibilizaram equações específicas para a espécie Manilkara spp. na área de manejo da FNT (RIBEIRO et al., 2014), avalições pontuais sobre a eficiência de equações específicas por Unidade de Produção Anual (UPA), bem como por classe diamétrica, ainda são inexistentes. Logo, são imprescindíveis para o aprimoramento das estimativas volumétricas da espécie na área de estudo e em outros locais da Amazônia. Assim, este estudo teve como objetivo avaliar a eficiência de equações específicas por UPA e por classe diamétrica, comparando-as com uma equação geral, para Manilkara spp., em área florestal sob manejo na FNT, estado do Pará.

\section{MATERIAL E MÉTODOS}

\section{1. Área de estudo}

O estudo foi realizado na FNT, Unidade de Conservação (UC) federal localizada no oeste do Estado do Pará (Figura 1), a qual margeia a Rodovia Santarém-Cuiabá (BR-163), abrangendo áreas dos municípios de Belterra, Aveiro, Placas e Rurópolis, entre os paralelos $2^{\circ} 45$ e $4^{\circ} 10^{\prime}$ de latitude sul e entre os meridianos $54^{\circ} 45^{\prime}$ e $55^{\circ} 30^{\prime}$ de longitude oeste. A UC ocupa uma área de aproximadamente 544.927 hectares e apresenta topografia variando de suavemente ondulada a ondulada, predominando o Latossolo Amarelo Distrófico e vegetação caracterizada como Floresta Ombrófila Densa (IBAMA, 2004). A concentração de chuvas ocorre entre janeiro e maio, com precipitação média anual de $1.820 \mathrm{~mm}$. O clima da região, de acordo com a reclassificação de Köppen, é do tipo Ami (quente e úmido), com temperatura média anual de $25,5^{\circ} \mathrm{C}$ (ALVARES et al., 2013).

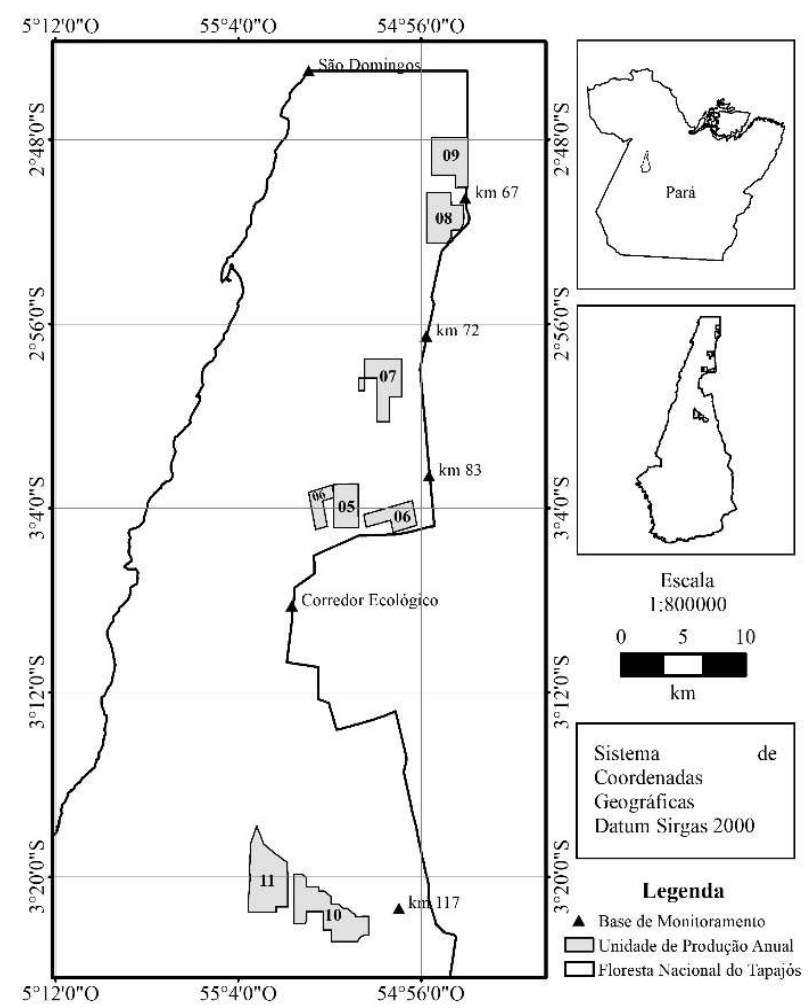

Figura 1. Localização da Floresta Nacional do Tapajós e das Unidades de Produção Anual distribuídas próximo às bases de monitoramento na Rodovia Cuiabá-Santarém (BR163), Pará, Brasil. Figure 1. Location of the Tapajós National Forest and the Annual Production Units (APUs) distributed near the forest monitoring bases along the Santarém-Cuiabá highway (BR163), Pará, Brazil.

\subsection{Obtenção e análise dos dados}

Os dados são provenientes dos Inventários Florestais à $100 \%$ e da cubagem rigorosa das árvores de Manilkara spp. com DAP (diâmetro medido à 1,3 $\mathrm{m}$ do solo) igual ou maior que $50 \mathrm{~cm}$, selecionadas para corte em sete UPAs, na área de manejo florestal da FNT. As UPAs estão em diferentes locais com acesso em pontos distintos ao longo da rodovia CuiabáSantarém (BR163): UPAs 05 e 06 (Km 83); UPA 07 (Km 72); UPAs 08 e 09 (Km 67); e UPAs 10 e 11 (Km 117) (Figura 1). Os dados foram obtidos junto à Cooperativa Mista da Flona do Tapajós (COOMFLONA), que possui concessão não onerosa para a execução do Manejo Florestal Comunitário na área.

As árvores-amostra utilizadas no ajuste dos modelos volumétricos, estão distribuídas nas sete UPAs e em sete classes de diâmetro (DAP) com amplitude de $10 \mathrm{~cm}$ (Tabela 1). O volume dito real das 5.231 árvores foi determinado até a altura comercial $\left(h_{c}\right)$ por meio do método de Smalian.

Quatro modelos volumétricos, dois de simples entrada e dois de dupla entrada (Tabela 2), foram inicialmente ajustados, por meio de regressão linear, para todo o conjunto de dados (5.231 árvores-amostra) de Manilkara spp. Foi selecionado o modelo com melhor desempenho na geração de uma equação geral representativa da espécie nas sete UPAs, bem como nas diferentes classes de DAP nas quais as árvores-amostra foram 
distribuídas. Os mesmos estão entre os principais modelos testados e selecionados na maioria dos estudos na Amazônia (THAINES et al., 2010; SANTOS et al., 2012; BARRETO et al., 2014; RIBEIRO et al., 2014; GIMENEZ et al., 2015; TONINI; BORGES, 2015; CYSNEIROS et al., 2017; LIMA, 2017).

Tabela 1. Número de árvores-amostra utilizadas para o ajuste de um modelo volumétrico geral e modelos específicos por Unidade de Produção Anual (UPA) e por classe de diâmetro (DAP), para Manilkara spp. em área de manejo florestal, na Floresta Nacional do Tapajós, Pará, Brasil.

Table 1. Number of sample trees used for the adjustment of one general volumetric model and specific volumetric models per Annual Production Unit (UPA) and per diameter class (DBH), for Manilkara spp. in forest management area, in the Tapajós National Forest, Pará, Brazil.

\begin{tabular}{ccccccccc}
\hline \multirow{2}{*}{ UPAs } & \multicolumn{7}{c}{ Centro de classe diamétrica (DAP cm) } & \multirow{2}{*}{ Total } \\
\cline { 2 - 7 } & 55 & 65 & 75 & 85 & 95 & 105 & $>110$ & \\
\hline 5 & 8 & 66 & 110 & 87 & 57 & 32 & 18 & 378 \\
6 & 29 & 143 & 167 & 102 & 72 & 27 & 20 & 560 \\
7 & 14 & 86 & 161 & 151 & 72 & 20 & 8 & 512 \\
8 & - & 17 & 53 & 33 & 15 & 19 & 7 & 144 \\
9 & 16 & 162 & 219 & 140 & 74 & 33 & 19 & 663 \\
10 & 33 & 129 & 373 & 247 & 163 & 66 & 39 & 1.050 \\
11 & 474 & 595 & 491 & 192 & 105 & 44 & 23 & 1.924 \\
\hline Total & 574 & 1.198 & 1.574 & 952 & 558 & 241 & 134 & 5.231 \\
\hline
\end{tabular}

Tabela 2. Modelos volumétricos testados para a obtenção de equação geral para Manilkara spp. em área de manejo floresta, na Floresta Nacional do Tapajós, Pará, Brasil

Table 2. Volumetric models tested to obtaining the general equation for Manilkara spp. in the management area of the Tapajós National Forest, Pará, Brazil

\begin{tabular}{lcc}
\hline $\mathrm{N}^{\mathrm{o}}$ & Autor & Modelo Estatístico \\
\hline 1 & Koperzky-Gehrhardt & $\mathrm{v}=b_{0}+b_{1} \mathrm{DAP}^{2}+\varepsilon_{\mathrm{i}}$ \\
2 & Husch & $\ln \mathrm{v}=b_{0}+b_{1} \operatorname{lnDAP}+\varepsilon_{\mathrm{i}}$ \\
3 & Spurr logaritmizado $(1952)$ & $\ln \mathrm{v}=b_{0}+b_{1} \ln \left(\mathrm{DAP}^{2} h_{c}\right)+\varepsilon_{\mathrm{i}}$ \\
4 & Schumacher-Hall $(1933)$ & $\ln \mathrm{v}=b_{0}+b_{1} \operatorname{lnDAP}+b_{2} \ln h_{c}+\varepsilon_{\mathrm{i}}$ \\
\hline $\mathrm{N}^{\mathrm{o}}=$ número do modelo; $\mathrm{v}=$ volume comercial com casca, em m $\mathrm{m}^{3} ; \mathrm{DAP}=$ \\
diâmetro à altura de $1,30 \mathrm{~m}$, em cm; $\mathrm{h}_{\mathrm{c}}=$ altura comercial, em m; $b_{0}, b_{1}$ e $b_{2}=$ \\
coeficientes de regressão a serem estimados; $\ln =\operatorname{logaritmo~neperiano;~e~} \varepsilon_{\mathrm{i}}=$ \\
erro aleatório.
\end{tabular}

Em seguida, o modelo selecionado foi ajustado especificamente para cada conjunto de dados estratificados por UPA e por classe de DAP, gerando 14 equações específicas. Para seleção da melhor equação geral, bem como para a avaliação da qualidade do ajuste das equações específicas, levou-se em consideração o maior coeficiente de determinação ajustado $\left(\mathrm{R}^{2}{ }_{\mathrm{aj}}\right)$, os menores valores de erro padrão de estimativa $\left(\mathrm{S}_{\mathrm{yx}}\right)$ absolutos e percentuais e a distribuição gráfica dos resíduos e o histograma de frequência dos resíduos, com objetivo de verificar possíveis tendências ao longo da linha de ajuste (CAMPOS; LEITE, 2017). Estes parâmetros foram calculados por meio das seguintes equações:

$$
\begin{aligned}
& \mathrm{R}^{2}{ }_{\mathrm{aj}}=1-\left(1-\mathrm{R}^{2}\right)\left(\frac{\mathrm{n}-1}{\mathrm{n}-\mathrm{k}}\right) \\
& \mathrm{S}_{\mathrm{yx}(\mathrm{m})}=\frac{\sqrt{\sum\left(\mathrm{vi}_{\mathrm{i}(\mathrm{obs})}-\mathrm{V}_{\mathrm{i}(\text { est }))^{2}}\right.}}{\mathrm{n}-\mathrm{k}} \\
& \mathrm{S}_{\mathrm{yx}} \%=\frac{\mathrm{S}_{\mathrm{yx}}}{\overline{\mathrm{Y}}} 100 \\
& \operatorname{Res}(\%)=\frac{\left(\mathrm{V}_{\mathrm{i}(\mathrm{est})}-\mathrm{V}_{\mathrm{i}(\mathrm{obs})}\right)}{\mathrm{V}_{\mathrm{i}(\mathrm{obs})}} 100
\end{aligned}
$$

em que: $\mathrm{R}^{2}{ }_{\text {aj }}=$ coeficiente de determinação ajustado; $\mathrm{R}^{2}=$ coeficiente de determinação; $\mathrm{n}=$ número de observações (árvores-amostra); $\mathrm{k}=$ número de coeficientes do modelo; $\mathrm{S}_{\mathrm{yx}}=$ erro padrão de estimativa; = $\mathrm{v}_{\mathrm{i}(\mathrm{obs})}=$ volume comercial observado da i-ésima árvore, em $\mathrm{m}^{3} ; \mathrm{v}_{\mathrm{i}(\mathrm{est})}$ $=$ volume comercial estimado da i-ésima árvore, $\mathrm{em}^{3} ; \overline{\mathrm{Y}}=$ média do volume comercial observado das árvores-amostra; Res (\%) = resíduos percentuais das estimativas.

Os modelos logarítmicos tiveram os erros padrão de estimativa recalculados, pois os valores obtidos pela análise de variância da regressão estavam na dimensão logarítmica. Os coeficientes dos modelos foram estimados pelo método dos mínimos quadrados, sendo aplicado o teste $\mathrm{t}(\alpha=0,05)$ para verificar a significância dos coeficientes da regressão.

A equação geral selecionada, bem como as equações específicas, foram validadas por meio do teste do Quiquadrado $\left(\chi^{2}\right)$, comparando os volumes observados com os volumes estimados. Para isso, foram utilizadas árvoresamostra independentes da base de dados utilizada para os ajustes, correspondendo a $10 \%$ do número de árvores presentes inicialmente em cada UPA e em cada classe de DAP. Para a validação da equação geral, os dados separados para a validação das equações específicas foram reunidos para, posteriormente, aplicar-se o teste.

Para verificar a existência de diferença estatística entre a equação geral e as equações específicas ajustadas para as UPAs e para as classes de DAP, adotou-se o teste de identidade de modelos proposto por Graybill (1976), indicando ou não a influência da localização das UPAs e da variação estrutural das árvores sobre as equações. O teste consiste, basicamente, na redução da soma dos quadrados, permitindo verificar estatisticamente, pelo teste de F (Tabela 3), a significância da diferença entre o total das somas dos quadrados das regressões ajustadas para cada UPA e para cada classe de DAP isoladamente (modelo completo), e a soma do quadrado da regressão ajustada para o conjunto total dos dados (modelo reduzido).

Tabela 3. Análise de variância para o teste de identidade de modelos. Table 3. Analysis of variance for the model identity test.

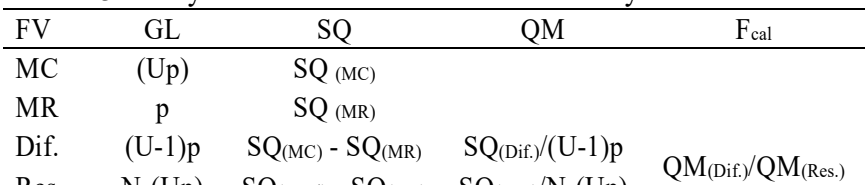

$\begin{array}{lllll}\text { Res. } & \mathrm{N}-(\mathrm{Up}) & \mathrm{SQ}_{(\text {(Total) }}-\mathrm{SQ}_{(\mathrm{MC})} & \mathrm{SQ}_{(\text {(Res. })} / \mathrm{N}-(\mathrm{Up}) & \mathrm{QM}_{(\text {(Dif.) }} / \mathrm{QM}_{(\text {Res. }}\end{array}$

Total N SQ Total

$\mathrm{FV}=$ fonte de variação; $\mathrm{MC}=$ modelo completo; $\mathrm{MR}=$ modelo reduzido; Dif. $=$ Diferença para testar hipótese; Res. = Resíduos; $\mathrm{GL}=$ grau de liberdade; $\mathrm{SQ}=$ soma dos quadrados; $\mathrm{QM}=$ quadrado médio; $\mathrm{F}=$ teste $\mathrm{F} ; \mathrm{U}=$ número de $\mathrm{UPAs}$ e de classes de DAP; $p$ = número de parâmetros do modelo reduzido; $\mathrm{N}=$ número de observações do modelo reduzido.

\section{As hipóteses testadas foram:}

$\mathrm{H}_{0}=$ a equação geral ajustada não apresenta diferença em relação às equações específicas ajustadas por UPA e por classe de DAP.

$\mathrm{H}_{1}=$ a equação geral ajustada apresenta diferença em relação às equações específicas ajustadas por UPA e por classe de DAP.

Ao não se rejeitar $\mathrm{H}_{0}$, conclui-se (para $\alpha=0,05$ ) que a equação geral não difere estatisticamente das equações específicas ajustadas para cada caso (UPAs e classes de DAP), assumindo-se que uma única equação pode representar o conjunto total de dados. Nos casos em que $\mathrm{H}_{0}$ foi rejeitada, foram realizados testes aos pares de UPAs e de classes de DAP 
para a identificação das equações específicas que diferem entre si, considerando-se, também, um nível de significância de 5\%.

\section{RESULTADOS}

3.1. Seleção de equação geral

Os coeficientes estimados na regressão e as estatísticas de ajuste e precisão para todo o conjunto de dados são apresentados na Tabela 4. Como esperado, as equações obtidas por meio dos modelos de dupla entrada (3 e 4) apresentaram desempenho superior, quando comparadas àquelas obtidas a partir dos modelos de simples entrada (1 e
2), em função da presença da altura comercial $\left(\mathrm{h}_{\mathrm{c}}\right)$ como varável auxiliar na explicação do volume. As estatísticas de ajuste e precisão indicaram que a inserção da altura comercial causou uma redução do erro de estimativa de, aproximadamente, $6 \%$. Os coeficientes de todas as equações foram estatisticamente significativos $(\mathrm{p} \leq 0,05)$ de acordo com o teste $\mathrm{t}$ da regressão linear.

$\mathrm{Na}$ Figura 2, são apresentadas a dispersão e os histogramas de distribuição dos resíduos para as equações ajustadas a partir dos modelos de Spurr e Shumacher-Hall, visto que resultaram em menores erro médios.

Tabela 4. Coeficientes e estatísticas das equações de volume comercial com casca ajustadas para Manilkara spp. em sete Unidades de Produção Anual, distribuídas em sete classes de DAP, em área de manejo florestal, na Florestal Nacional do Tapajós, Pará, Brasil.

Table 4. Coefficients and statistics of equations of commercial volume with bark adjusted based on sample trees of Manilkara spp. in seven Annual Production Units, distributed in seven classes of DBH, in forest management area, in the National Forest of Tapajós, Pará, Brazil.

\begin{tabular}{|c|c|c|c|c|c|c|c|}
\hline & \multirow{2}{*}{ Modelos } & \multicolumn{3}{|c|}{ Coeficientes da regressão } & \multirow{2}{*}{$\mathrm{R}^{2}$ aj } & \multirow{2}{*}{$\mathrm{S}_{\mathrm{yx}}$} & \multirow{2}{*}{$\mathrm{S}_{\mathrm{yx}}(\%)$} \\
\hline & & $b_{0} \wedge$ & $b_{1} \wedge$ & $b_{2} \wedge$ & & & \\
\hline 1 & Koperzky-Gehrhardt & $0,55700^{*}$ & $0,00084^{*}$ & & 0,71 & 1,28 & 22,58 \\
\hline 2 & Husch & $-5,92642 *$ & $1,75173^{*}$ & & 0,69 & 1,28 & 22,76 \\
\hline 3 & Spurr (logaritimizado) & $-7,95037^{*}$ & $0,82380 *$ & & 0,84 & 0,93 & 16,61 \\
\hline 4 & Schumacher-Hall & $-7,94536^{*}$ & $1,58930^{*}$ & $0,90617^{*}$ & 0,84 & 0,93 & 16,65 \\
\hline
\end{tabular}

$\mathrm{R}^{2}{ }_{\text {aj }}=$ coeficiente de determinação ajustado; $S_{\mathrm{yx}}=$ erro padrão da estimativa absoluto, em $\mathrm{m}^{3} ; \mathrm{S}_{\mathrm{yx}}(\%)=$ erro padrão da estimativa em porcentagem; $b_{0} \wedge, b_{1} \wedge \mathrm{e} b_{2} \wedge=$ coeficientes dos modelos; ${ }^{*}$ Coeficiente significativo, de acordo com o teste $\mathrm{t}(\mathrm{p} \leq 0,05)$.
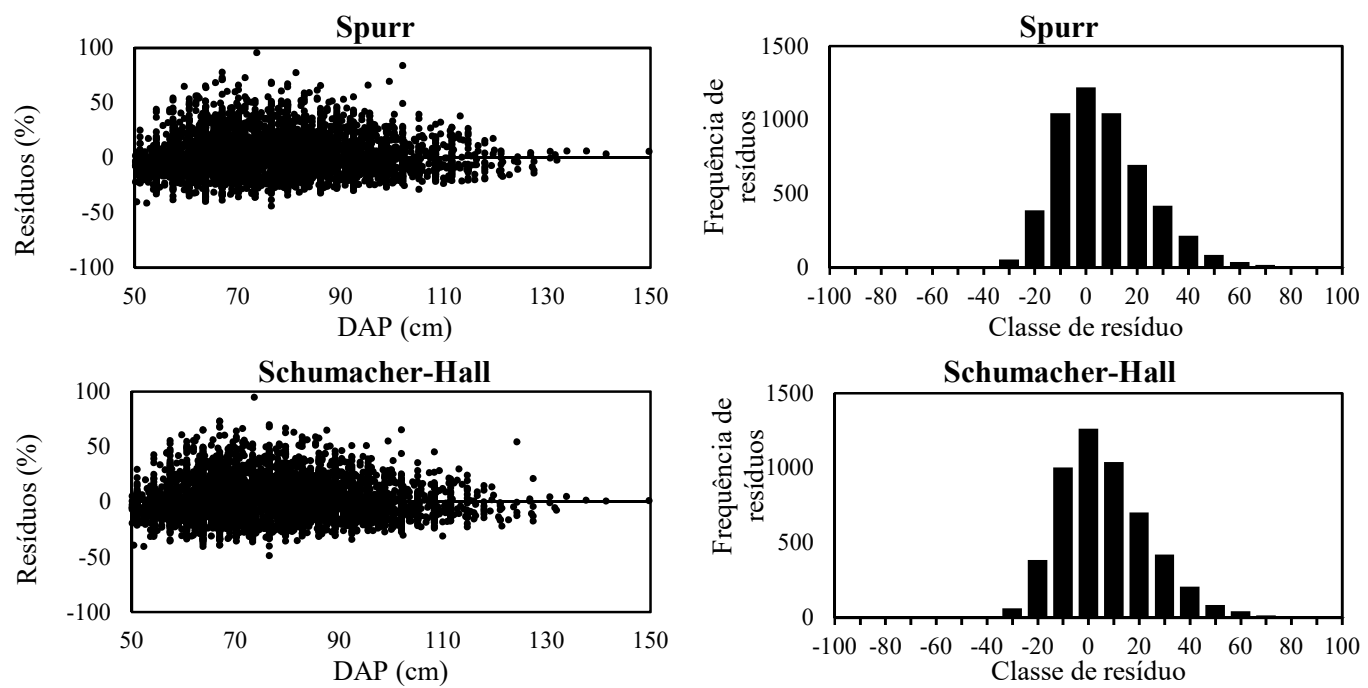

Figura 2. Análise gráfica e histograma dos resíduos para os modelos ajustados de melhor desempenho com base na amostra total de Manilkara spp. distribuída em sete classes de DAP, nas sete Unidades de Produção Anual, em área de manejo florestal, na Florestal Nacional do Tapajós, Pará, Brasil.

Figure 2. Graphical analysis and histogram of the residuals for the adjusted models better performance based on the total sample of Manilkara spp. distributed in seven classes of DBH, in the seven Annual Production Units, in forest management area, in the National Forest of Tapajós, Pará, Brazil.

Observa-se que, apesar da tendência em superestimar os volumes, tais modelos apresentaram desempenho aceitável, considerando a heterogeneidade das florestas nativas amazônicas, até mesmo entre árvores de mesma espécie. Ambas apresentaram distribuições dos resíduos semelhantes, principalmente para as árvores com menores diâmetros.

Contudo, a partir de $120 \mathrm{~cm}$ de DAP, observa-se que a equação ajustada a partir do modelo de Spurr apresentou desempenho superior, em relação a equação de SchumacherHall, uma vez que apresentou erros distribuídos de forma mais homogênea ao longo da linha zero e com menores tendências em superestimar os volumes das maiores árvores, apesar de pouco perceptível. Assim, o modelo de Spurr foi selecionado para o ajuste e obtenção das equações específicas para as UPAs e classes de DAP. Após a seleção da equação de Spurr, aplicou-se o teste Qui-quadrado $\left(\chi^{2}\right)$ para validação da mesma. Constatou-se não haver diferença significativa entre os valores observados e estimados do volume, a um nível de $95 \%$ de probabilidade. $\mathrm{O}$ valor calculado do teste $\left(\chi_{\text {calculado }}^{2}=248,43\right)$ foi menor que o valor tabelado $\left(\chi_{\text {tabelado }}^{2}=554,18\right)$, indicando que a equação geral selecionada é válida para as estimativas volumétricas de Manilkara spp.

3.2. Equações específicas por Unidade de Produção Anual (UPA)

Uma vez selecionado para a amostra total dos dados, o de modelo Spurr foi ajustado com base nos dados estratificados por UPA, gerando sete equações específicas, onde os resultados dos ajustes são apresentados na Tabela 5. As estatísticas de ajuste e precisão indicaram melhor desempenho 
das equações específicas em relação a equação geral, mostrando que as mesmas são mais adequadas para estimar os volumes comerciais de Manilkara spp. nas unidades de manejo na FNT.

$\mathrm{O}$ teste de identidade de modelos foi aplicado, constatando-se diferença estatística entre a equação geral e as equações específicas $\left(\mathrm{F}_{\mathrm{cal}}=220,65 ; p=0,0000\right)$, levando a rejeitar a hipótese $\mathrm{H}_{0}$ ao nível de $5 \%$ de significância. Assim, verificou-se que não é adequado utilizar a equação geral para a estimativa dos volumes comerciais da espécie nas sete UPAs. A partir deste resultado, seguiu-se, então, para os testes aos pares de UPAs (21 combinações) para definição de quais equações específicas diferem entre si. Os resultados dos testes aos pares são apresentados na Tabela 6 , onde constata-se que a hipótese de igualdade estatística entre as equações $\left(\mathrm{H}_{0}\right)$ foi aceita apenas entre as UPAs 05 e 09; 06 e 08; e, 07 e 10, ou seja, apenas nesses casos seria estatisticamente adequado utilizar uma equação geral para representar ambas as UPAs.

Tabela 5. Coeficientes e estatísticas da equação de volume geral e das equações específicas por Unidade de Produção Anual (UPA), ajustadas com o modelo de Spurr, para Manilkara spp., em área de manejo florestal, na Florestal Nacional do Tapajós, Pará, Brasil.

Table 5. Coefficients and statistics of the general volume equation and the specific equations by Annual Production Unit (UPA), adjusted from the Spurr model, for Manilkara spp., in forest management area, in the National Forest of Tapajós, Pará, Brazil.

\begin{tabular}{|c|c|c|c|c|c|}
\hline \multirow{2}{*}{ UPAs } & \multicolumn{2}{|c|}{ Coeficientes da } & \multirow{2}{*}{$\mathrm{R}^{2} \mathrm{aj}$} & \multirow{2}{*}{$\mathrm{S}_{\mathrm{yx}}$} & \multirow{2}{*}{$\mathrm{S}_{\mathrm{yx}}(\%)$} \\
\hline & $b_{0} \wedge$ & $b_{1} \wedge$ & & & \\
\hline Geral & $-7,95037$ & 0,82380 & 0,84 & 0,93 & 16,61 \\
\hline 05 & $-9,30443^{*}$ & $0,92914 *$ & 0,86 & 0,91 & 15,83 \\
\hline 06 & $-8,94840 *$ & $0,90173 *$ & 0,89 & 0,82 & 14,84 \\
\hline 07 & $-8,69007^{*}$ & $0,88446^{*}$ & 0,89 & 0,72 & 12,75 \\
\hline 08 & $-9,06647 *$ & $0,91231^{*}$ & 0,88 & 0,93 & 14,81 \\
\hline 09 & $-8,77720 *$ & $0,88371^{*}$ & 0,87 & 0,76 & 15,57 \\
\hline 10 & $-8,85525^{*}$ & $0,89868^{*}$ & 0,87 & 0,90 & 14,49 \\
\hline 11 & $-8,15651^{*}$ & $0,85183 *$ & 0,91 & 0,68 & 12,46 \\
\hline
\end{tabular}

$\mathrm{R}_{\text {aj }}^{2}=$ coeficiente de determinação ajustado; $S_{\mathrm{yx}}=$ erro padrão da estimativa absoluto, em m ${ }^{3} ; \mathrm{S}_{\mathrm{yx}}(\%)=$ erro padrão da estimativa em porcentagem; $b_{0} \wedge$, e $b_{1}^{\wedge}=$ coeficientes dos modelos; $*$ Coeficiente significativo, de acordo com o teste $\mathrm{t}(\mathrm{p} \leq 0,05)$.

Tabela 6. Resultados ( $p$-valor) dos testes de identidade de modelos realizados aos pares para comparação das equações específicas por Unidade de Produção Anual (UPA) para Manilkara spp., em área de manejo florestal, na Florestal Nacional do Tapajós, Pará, Brasil.

Table 6. Results ( $p$-value) of the model identity tests performed in pairs to compare the specific equations by Annual Production Unit (UPA) for Manilkara spp., in forest management area, at the National Forest of Tapajós, Pará, Brazil.

\begin{tabular}{ccccccc}
\hline UPAs & 06 & 07 & 08 & 09 & 10 & 11 \\
\hline 05 & 0,0024 & 0,0000 & 0,0352 & 0,1343 & 0,0000 & 0,0000 \\
06 & - & 0,0000 & 0,8236 & 0,0000 & 0,0000 & 0,0000 \\
07 & - & - & 0,0007 & 0,0000 & 0,7335 & 0,0000 \\
08 & - & - & - & 0,0015 & 0,0006 & 0,0000 \\
09 & - & - & - & - & 0,0000 & 0,0000 \\
10 & - & - & - & - & - & 0,0000 \\
\hline
\end{tabular}

As equações específicas geradas para as sete UPAs foram submetidas ao processo de validação por meio do teste Quiquadrado $\left(\chi^{2}\right)$, comparando os volumes observados com os volumes estimados. Assim, constatou-se não haver diferença significativa entre os volumes, a um nível de 95\% de probabilidade, uma vez que os valores de $\chi^{2}$ calculados foram menores que os valores de $\chi^{2}$ tabelados, indicando que as equações específicas são válidas para as estimativas volumétricas de Manilkara spp. nas UPAs.

3.3. Equações específicas por classe de diâmetro (DAP)

O modelo de Spurr também foi ajustado especificamente para cada classe de DAP, gerando sete equações específicas, apresentadas na Tabela 7. Com exceção da equação ajustada para a classe $3(70,0-79,9)$, a qual apresentou o maior número de árvores em relação as demais classes, todas as equações específicas resultaram em menores erros percentuais de estimativa, quando comparadas à equação geral, indicando a melhora na precisão das estimativas. Observa-se, também, que as três primeiras classes apresentaram erros absolutos menores em relação as quatro últimas classes.

Tabela 7. Coeficientes e estatísticas da equação geral e das equações específicas por classe de diâmetro (DAP), ajustadas com o modelo de Spurr, para Manilkara spp., em área de manejo florestal, na Florestal Nacional do Tapajós, Pará, Brasil.

Table 7. Coefficients and statistics of the general equation (all classes) and of the specific equations by diameter class (DBH), adjusted from the Spurr model, for Manilkara spp., in forest management area, in the National Forest of Tapajós, Pará, Brazil.

\begin{tabular}{|c|c|c|c|c|c|}
\hline \multirow{2}{*}{ Classe de DAP } & \multicolumn{2}{|c|}{ Coeficientes da regressão } & \multirow{2}{*}{$\mathrm{R}^{2}$ aj } & \multirow{2}{*}{$\mathrm{S}_{\mathrm{yx}}$} & \multirow{2}{*}{$\begin{array}{l}S_{y x} \\
(\%)\end{array}$} \\
\hline & $b_{0} \wedge$ & $b_{1} \wedge$ & & & \\
\hline Geral & $-7,95037$ & 082380 & 0,84 & 0,93 & 16,61 \\
\hline & 680 & & & 0,45 & 14,18 \\
\hline & 8,500 & $8 / 2$ & & 0,57 & 14,23 \\
\hline & 8.91 & * & & 0,93 & 17,63 \\
\hline & 58 & & & 1,03 & 15,83 \\
\hline $5(9$ & $-8,71$ & $0,88661 *$ & & 1,18 & 14,90 \\
\hline $6(100,0-109,9)$ & $-8,3813$ & $086055 *$ & & 1,24 & 13,06 \\
\hline $7(\geq 110,0)$ & $-9,08188^{*}$ & $0,91518 *$ & 0,74 & 1,33 & 11,61 \\
\hline
\end{tabular}

$\mathrm{R}^{2}{ }_{\mathrm{aj}}=$ coeficiente de determinação ajustado; $\mathrm{S}_{\mathrm{yx}}=$ erro padrão da estimativa absoluto, em m ${ }^{3} ; \mathrm{S}_{\mathrm{yx}}(\%)=$ erro padrão da estimativa em porcentagem; $b_{0} \wedge$, e $b_{1}^{\wedge}=$ coeficientes dos modelos; $*$ Coeficiente significativo, de acordo com o teste $\mathrm{t}(\mathrm{p} \leq 0,05)$.

Aplicou-se, então, o teste de identidade e a hipótese de que as equações são estatisticamente iguais $\left(\mathrm{H}_{0}\right)$ foi rejeitada $\left(\mathrm{F}_{\mathrm{cal}}\right.$ $=7,38 ; p=0,0000)$, ou seja, não é adequado generalizar uma equação comum para todas as classes de DAP. Foi então necessário realizar testes aos pares de classes para identificar as classes diferentes, onde os resultados são apresentados na Tabela 8

Tabela 8. Resultados ( $p$-valor) dos testes de identidade de modelos realizados aos pares para comparação das equações específicas por classes de DAP para Manilkara spp., em área de manejo florestal, na Florestal Nacional do Tapajós, Pará, Brasil.

Table 8. Results ( $p$-value) of the model identity tests performed in pairs to compare the specific equations by classes of DBH for Manilkara spp., in forest management area, at the National Forest of Tapajós, Pará, Brazil.

\begin{tabular}{ccccccc}
$\begin{array}{c}\text { Classe de } \\
\text { DAP }\end{array}$ & 2 & 3 & 4 & 5 & 6 & 7 \\
\hline 1 & 0,0000 & 0,0000 & 0,0001 & 0,0011 & 0,4183 & 0,0723 \\
2 & - & 0,0007 & 0,0059 & 0,0429 & 0,6196 & 0,2302 \\
3 & - & - & 0,0242 & 0,0541 & 0,3858 & 0,1078 \\
4 & - & - & - & 0,9550 & 0,5607 & 0,7158 \\
5 & - & - & - & - & 0,5251 & 0,6831 \\
6 & - & - & - & - & - & 0,3107 \\
\hline
\end{tabular}

Classe de DAP: 1 (50,0-59,9); 2 (60,0-69,9); 3 (70,0-79,9); 4 (80,0-89,9); 5 $(90,0-99,9) ; 6(100,0-109,9)$; e $7(\geq 110,0)$, em cm. 
Observa-se que na maioria das combinações a hipótese nula foi aceita $(p \geq 0,05)$, principalmente nas combinações entre as maiores classes. De modo geral, as combinações onde a hipótese nula foi rejeitada foram entre as duas primeiras classes e as classes subsequentes, justificando-se pela diferença estrutural entre as árvores presentes em classes menores e maiores, que pode gerar equações significativamente diferentes.

O teste Qui-quadrado $\left(\chi^{2}\right)$ também foi aplicado para validação das equações específicas ajustadas para cada classe de DAP, constatando-se não haver diferença significativa entre os volumes observados e estimados a um nível de $95 \%$ de probabilidade (valores de $\chi^{2}$ calculados $\leq$ valores de $\chi^{2}$ tabelados), ou seja, as equações são aderentes aos conjuntos específicos de dados. Logo, as equações específicas ajustadas são válidas para as estimativas volumétricas de Manilkara spp. nas diferentes classes diamétricas na área de manejo da FNT.

\section{DISCUSSÃO}

Por representarem melhor o volume das árvores, os modelos de dupla entrada são normalmente os mais utilizados nas estimativas volumétricas, tanto em plantios florestais quanto nas florestas naturais (LEITE; ANDRADE, 2003). Obviamente, os modelos de Spurr e Schumacher-Hall têm como entrada a altura comercial, a qual exige esforço adicional na medição das árvores. Todavia, pela performance superior desses modelos, deve-se buscar aprimorar técnicas que facilitem e melhorem as medições dessa variável, minimizando desta forma, o erro na estimativa dos volumes comerciais. Apesar de as equações apresentarem coeficientes de determinação ajustados abaixo de $85 \%$ e erro padrão das estimativas superiores a $15 \%$, ressalta-se que são resultados aceitáveis considerando o comportamento estrutural variável das árvores de espécies nativas amazônicas.

A altura comercial das árvores nas florestas nativas amazônicas é, na maioria dos casos, obtida por meio de estimativas. Assim, como relatado por Thaines et al. (2010) e Batista et al. (2014), a presença de vários estratos arbóreos nas florestas tropicais e, em muitos casos, a inexperiência dos responsáveis por realizarem as estimativas dificultam a obtenção desta variável, o que reflete diretamente na incerteza de suas estimativas, e consequentemente nas estimativas volumétricas. Portanto, é necessário cautela na medição e inserção da altura comercial nos modelos volumétricos, a fim de minimizar os erros nas predições (CYSNEIROS et al., 2017).

Como concluído por Gomes et al. (2018), é necessário investir no aperfeiçoamento das técnicas de estimativa da altura comercial e em treinamento dos trabalhadores envolvidos no inventário. De acordo com esses autores, quando se considera o mínimo de erro na obtenção da altura comercial, o volume estimado corresponde a mais de $95 \%$ do volume observado, indicando que, se maior rigor for empreendido na obtenção das medidas no inventário, estimativas volumétricas com precisão aceitável podem ser geradas com base no DAP e na altura comercial.

Nesse contexto, por se ajustar melhor aos dados de Manilkara spp., nesse estudo o modelo de Spurr foi selecionado para a obtenção da equação geral. O mesmo já foi recomendado em outros estudos realizados na Amazônia, como apresentado por Cysneiros et al. (2017). Segundo Andrade (2017), dentre os oito modelos volumétricos mais avaliados no Brasil nos últimos anos, o modelo de Spurr foi selecionado em 14\% dos estudos, ocupando a segunda posição, atrás apenas do modelo de Schumacher-Hall.

Na FNT, Ribeiro et al. (2014) e Silva et al. (2017) também indicaram em seus estudos que a equação geral obtida com base no modelo de Spurr foi adequada e, portanto, recomendada para as estimativas volumétricas das espécies comerciais. Ribeiro et al. (2014) testaram também a eficiência de equações especificamente para Manilkara spp. na FNT e observaram que a equação de Spurr, bem como a SchumacherHall, foi adequada para as estimativas de volume comercial na ocasião.

Quando as equações foram geradas especificamente para as diferentes UPAs e classes diamétricas, foram observados menores erros de estimativas em relação a equação geral, sugerindo que não é adequado a utilização de uma equação geral para Manilkara spp. em todas as unidades manejadas anualmente e para todas as árvores em diferentes classes de DAP, apesar de o teste Qui-quadrado ter indicado a validade da mesma. Essas observações foram corroboradas pelo teste de identidade de modelos, onde verificou-se que uma única equação não deveria ser utilizada para todas as UPAs, bem como para todas as classes de diâmetro, uma vez que este procedimento não é adequado estatisticamente.

As condições variáveis de clima, solo e relevo, bem como a disponibilidade hídrica, influenciam o crescimento e a relação entre as variáveis dendrométricas das árvores (SOARES et al., 2011; VALENTE et al., 2011; SANTOS et al., 2012). Além disso, o tipo de solo também é um dos principais fatores que determina a estrutura da floresta (LOURES et al., 2007; SOUZA et al., 2006), logo, influencia diretamente as relações dendrométricas. O tipo de solo predominante na FNT é o Latossolo Amarelo Distrófico. Contudo, avaliações realizadas em áreas mais específicas na área identificaram subvariações nos tipos de solo (IBAMA, 2004). De acordo com Martins et al. (2015), a interação entre esses fatores pode proporcionar o crescimento distinto das variáveis das árvores entre os locais, culminando em volumes diferentes entre os mesmos.

Na FNT, é perceptível a variação das características como solo e topografia, principalmente no sentido Norte-Sul, no qual as UPAs estão distribuídas espacialmente. Espírito-Santo et al. (2005) verificaram que existem pelo menos três padrões fitogeográficos distintos na FNT. Neste cenário, equações desenvolvidas a partir de conjuntos de dados provenientes de áreas de floresta com menor heterogeneidade, como UPAs, certamente devem gerar resultados mais precisos nas estimativas de volume. Gomes et al. (2018) enfatizaram a necessidade de utilização de equações específicas ao observarem que o emprego de uma equação geral levou à maximização do erro de estimativa volumétrica na FNT.

Quando se trata especificamente das equações avaliadas para as classes diamétricas, as estatísticas de precisão dos ajustes indicaram que as equações específicas ajustadas para as três primeiras classes apresentaram erros absolutos menores em relação as equações para as classes maiores. Além disso, os resultados do teste de identidade indicaram que as combinações das equações ajustadas para as classes menores foram, na maioria dos casos, estatisticamente diferentes das equações ajustadas para as classes subsequentes.

Essas observações sugerem que, caso equações específicas para classes em amplitudes menores (como $10 \mathrm{~cm}$, por exemplo) não sejam utilizadas, é importante que no mínimo duas equações sejam ajustadas, estratificando-se as árvores em 
duas classes, a fim de que estimativas mais acuradas sejam obtidas. Este procedimento torna-se importante principalmente porque as árvores maiores geralmente comportam os maiores erros associados às estimativas, uma vez que as variáveis dendrométricas que explicam o volume, nesses casos, são mais difíceis de serem obtidas (especialmente a altura comercial). Com esse resultado, presume-se, também, que se deve buscar maior acurácia nas estimativas volumétricas das árvores de maior porte, principalmente no que diz respeito à medição do DAP e da altura comercial no inventário, uma vez que o potencial volumétrico das mesmas é maior e, consequentemente, seu valor econômico.

Como observado também por Cyneiros et al. (2017), equações específicas por classe diamétrica proporcionam maior precisão nas estimativas de volume. Todavia, como foi enfatizado por esses autores, a estratificação de dados para o ajuste de modelos volumétricos ainda não é comum na área florestal, principalmente nas florestas tropicais amazônicas, tornando as investigações e os resultados como os do presente estudo de extrema importância para o manejo das espécies comerciais nativas, notadamente quando se considera a maior importância comercial de determinadas espécies, como é o caso da Manilkara spp.

\section{CONCLUSÕES}

Não é adequado a utilização de uma equação geral para a estimativa dos volumes comerciais de Manilkara spp. em todas as UPAs na área de manejo da FNT, assim como em todas as classes diamétricas. As equações específicas, ajustadas a partir do modelo de Spurr logaritmizado, são válidas e recomendadas para as predições da produção volumétrica da espécie, cabendo ao gestor florestal decidir se o ganho em precisão justifica economicamente a utilização das mesmas.

\section{AGRADECIMENTOS}

Os autores agradecem à Cooperativa Mista da Flona Tapajós (COOMFLONA) por contribuir com a presente pesquisa.

\section{REFERÊNCIAS}

ALVARES, C. A.; STAPE, J. L.; SENTELHAS, P. C.; GONÇALVES, J. L. M.; SPAROVEK G. Koppen's climate classification map for Brazil. Meteorologische Zeitschrift, Berlin, v. 22, n. 6, p. 711-728, 2013. DOI: https://dx.doi.org/10.1127/0941-2948/2013/0507

ANDRADE, V. C. L. Modelos volumétricos de dupla entrada para aplicar em povoamentos florestais brasileiros. Floresta e Ambiente, Seropédica, v. 24, p. 1-10, 2017 DOI: http://dx.doi.org/10.1590/2179-8087.135415

BARRETO, W. F.; LEÃO, F. M.; MENEZES, M. C.; SOUZA, D. V. Equação de volume para apoio ao manejo comunitário de empreendimento florestal em Anapu, Pará. Pesquisa Florestal Brasileira, Colombo, v. 34, n. 80, p. 21-329, 2014. DOI: http://dx.doi.org/10.4336/2014.pfb.34.80.721

BARROS, P. L. C.; SILVA JÚNIOR, A. T. Equação de volume para árvores de uma floresta tropical densa no município de Anapu, Oeste do estado do Pará, Amazônia oriental. Revista de Ciências Agrárias, Belém, v. 51, n. 1, p.115-126, 2009.
BATISTA, J. L. F.; COUTO, H. T. Z.; SILVA FILHO, D. F. Quantificação de recursos florestais: árvores, arvoredos e florestas. 1. ed. São Paulo: Oficina de Textos, 2014. $384 \mathrm{p}$.

CAMPOS, J. C. C.; LEITE, H. G. Mensuração florestal: Perguntas e respostas. 5. ed. Editora UFV, 2017. 636 p.

CASTRO, T. C.; CARVAlHO, J. O. P. Dinâmica da população de Manilkara huberi (Ducke) A. Chev. durante 26 anos após a exploração florestal em uma área de terra firme na Amazônia brasileira. Ciência Florestal, Santa Maria, v. 24, n. 1, p. 161-169, 2014. DOI: http://dx.doi.org/10.5902/1980509813333

CYSNEIROS, V. C.; PELISSARI, A. L.; MACHADO, S. A.; FIGUEIREDO FILHO, A.; SOUZA, L. Modelos genéricos e específicos para estimativa do volume comercial em uma floresta sob concessão na Amazônia. Scientia Forestalis, Piracicaba, v. 45, n. 114, p. 295-304, 2017. DOI: http://dx.doi.org/10.18671/scifor.v45n114.06

ESPÍRITO-SANTO, F. D. B.; SHIMABUKURO, Y. E.; ARAGÃO, L. E. O. C.; MACHADO, E. L. M. Análise da composição florística e fitossociológica da Floresta Nacional do Tapajós com o apoio geográfico de imagens de satélite. Acta Amazonica, Manaus, v. 35, n. 2, p. $155-$ 173, 2005. DOI: http://dx.doi.org/10.1590/S004459672005000200006

GAMA, J. R. V.; SOUZA, A. L.; VIEIRA, D. S.; LEITE, H. G. Equações de volume para uma floresta ombrófila aberta, município de Codó, estado do Maranhão. Revista Brasileira de Ciências Agrárias, Recife, v. 12, n. 4, p. 535-542, 2017.2 DOI: http://dx.doi.org/10.5039/agraria.v12i4a5489

FIGUEIREDO FILHO, A. Estudos de modelos matemáticos para estimar o volume por unidade de área em uma floresta tropical úmida na Amazônia brasileira. 1983. 176f. Dissertação (Mestrado em Ciências) - Universidade Federal do Paraná, Curitiba, 1983.

GIMENEZ, B. O.; DANIELLI, F. E.; OLIVEIRA, C. K. A.; SANTOS, J.; HIGUCHI, N. Equações volumétricas para espécies comerciais madeireiras do sul do estado de Roraima. Scientia Forestalis, Piracicaba, v. 43, n. 106, p. 291-301, 2015.

GOMES, K. M. A.; SILVA-RIBEIRO, R. B.; GAMA, J. R. V.; ANDRADE, D. F. C. Eficiência na estimativa volumétrica de madeira na Floresta Nacional do Tapajós. Nativa, Sinop, v. 6, n. 2, p. 170-176, 2018. DOI: http://dx.doi.org/10.31413/nativa.v6i2.5237

GRAYBILL, F. A. Theory and application of the linear model. Massachusetts: Ouxburg Press, 1976. 704 p.

IBAMA_INSTITUTO BRASILEIRO DO MEIO AMBIENTE E DOS RECURSOS NATURAIS RENOVÁVEIS. Plano de Manejo da Floresta Nacional do Tapajós. Brasília, 2004. Disponível em: $<$ http://www.icmbio.gov.br.> Acessado em: 14 nov. 2018.

LEITE, H. G.; ANDRADE, V. C. L. Importância das variáveis altura dominante e altura total em equações hipsométricas e volumétricas. Revista Árvore, Viçosa, v. 27, n. 3, p. 341-348, 2003. DOI: http://dx.doi.org/10.1590/S010067622003000300005

LIMA, R. B. Produção volumétrica bruta, rendimento e modelagem de madeira serrada de espécies comerciais da Amazônia. 2017. 142f. Tese (Doutorado em Ciências Florestais) - Universidade Federal Rural do Pernambuco, Recife, 2017. 
LOURES, L.; CARVALHO, D. A.; MACHADO, E. L. M.; MARQUES, J. J. G. S. M. Florística, estrutura e características do solo de um fragmento de floresta paludosa no sudeste do Brasil. Acta Botanica Brasilica, São Paulo, v. 21, n. 4, p. 885-896, 2007. DOI: http://dx.doi.org/10.1590/S0102-33062007000400013

MARTINS, R. M.; LEITE, M. V. S.; CABACINHA, C. D.; ASSIS, A. L. Teste de identidade de modelos volumétricos para povoamentos de Eucalyptus sp. em sete municípios de Minas Gerais. Enciclopédia Biosfera, Goiânia, v. 11, n. 21, p. 1818-1833, 2015.

RIBEIRO, J. Avaliação ambiental econômica da produção de madeira de espécie nativa em dois municípios na Amazônia brasileira. 2008. 42f. Dissertação (Mestrado em Saúde Ambiental) - Universidade de São Paulo, São Paulo, 2008.

RIBEIRO, R. B. S.; GAMA, J. R. V.; MELO, L. O. Seccionamento para cubagem e escolha de equações de volume para a Floresta Nacional do Tapajós. Cerne, Lavras, v. 20, n. 4, p. 605-612, 2014. DOI: http://dx.doi.org/10.1590/01047760201420041400

SANTOS, A. T.; MATTOS, P. P.; BRAZ, E. M.; ROSOT, N. C. Equação de volume e relação hipsométrica em plantio de Ocotea porosa. Pesquisa Florestal Brasileira, Colombo, v. 32, n. 69, p. 13-21, 2012. DOI: http://dx.doi.org/10.4336/2012.pfb.32.69.13
SILVA, R. C.; MELO, L. O.; XIMENES, L. C.; SILVA, R. B. Ajuste de equações volumétricas para diferentes seccionamentos de tora em uma floresta sob manejo florestal comunitário. Acta Tecnológica, v. 12, n. 2, p. 85101, 2017.

SOARES, C. P. B.; PAULA NETO, F.; SOUSA, A. L. Dendrometria e inventário Florestal. UFV, 2011. 276 p. SOUZA, J. P.; ARAÚJO, G. M.; HARIDASAN, M. Influence of soil fertility on the distribution of tree species in a deciduous forest in the Triângulo Mineiro region of Brazil. Plant Ecology, Dordrecht, v. 191, n. 2, p. 253-263, 2006. DOI: https://dx.doi.org/10.1007/s11258-006-9240-2

THAINES, F.; BRAZ, E. M.; MATTOS, P. P.; THAINES, A. A. R. Equações para estimativa de volume de madeira para a região da bacia do Rio Ituxi, Lábrea, AM. Pesquisa Florestal Brasileira, Colombo, v. 30, n. 64, p. 283-289, 2010. DOI: http://dx.doi.org/10.4336/2010.pfb.30.64.283

TONINI, H.; BORGES, R. A. Equação de volume para espécies comerciais em Floresta Ombrófila Densa no sul de Roraima. Pesquisa Florestal Brasileira, Colombo, v. 35, n. $82, \quad$ p. $1-8, \quad 2015$. DOI: http://dx.doi.org/10.4336/2015.pfb.35.82.738

VALENTE, M. D. R.; QUEIROZ, V. T.; PINHEIRO, J. G.; MONTEIRO, L. A. S. Modelo de predição para o volume total de Quaruba (Vochysia inundata Ducke) via análise de fatores e regressão. Revista Árvore, Viçosa, v. 35, n. 2, p. 307-317, 2011. DOI: http://dx.doi.org/10.1590/S010067622011000200015 